\title{
INVESTIGACIÓN
}

Recibido: 04/11/2021 --- Aceptado:02/12/2021 --- Publicado: 03/01/2022

\section{IMPACTO DE LAS FAKE NEWS EN ESTUDIANTES DE PERIODISMO Y COMUNICACIÓN AUDIOVISUAL DE LA UNIVERSIDAD CARLOS III DE MADRID}

\section{Impact of fake news on students of journalism and audiovisual communication at Carlos III university of Madrid}

Eva Herrero Curiel. Universidad Carlos III de Madrid. España. eherrero@hum.uc3m.es

Patricia González Aldea. Universidad Carlos III de Madrid. España. patricia.gonzalez.aldea@uc3m.es

\section{Cómo citar el artículo:}

Herrero Curiel, E., y González Aldea, P. (2022). Impacto de las fake news en estudiantes de periodismo y comunicación audiovisual de la universidad Carlos III de Madrid. Vivat Academia. Revista de Comunicación, 155, 1-21. http:// doi.org/10.15178/va.2022.155.e1415

\section{RESUMEN}

En el actual contexto mediático cada vez son más las voces que alertan sobre la desinformación, las fake news o las noticias falsas y el impacto que éstas tienen a través de las redes sociales en jóvenes. Conocer en qué grado estudiantes de Periodismo detectan e interactúan con este tipo de información es necesario para articular estrategias dentro de los propios estudios que capaciten a futuros profesionales a detectar y evitar la propagación de piezas desinformativas. A partir de una triangulación metodológica se han obtenidos datos cuantitativos, mediante un cuestionario online administrado a una muestra de 200 estudiantes matriculados en el curso académico (2019/2020) en los grados del área de Periodismo. Y a través de dos focus group con estudiantes de estas titulaciones se han obtenido datos cualitativos sobre la opinión de los futuros periodistas acerca de las fake news. A pesar de que casi tres de cada cuatro estudiantes afirman saber distinguir una noticia falsa de una verdadera y más de la mitad consideran que el profesorado les ha facilitado herramientas para luchar contra la desinformación, el 97,6\% considera importante potenciar la alfabetización mediática desde las aulas universitarias.

PALABRAS CLAVE: Periodismo-alfabetización mediática-noticias falsas- 
desinformación-universitarios españoles.

\begin{abstract}
In the current media context more and more voices warn about disinformation, fake news or false news and the impact that these have through social media on young people. It is important to know to what degree Journalism students detect and interact with this type of information to design strategies within the studies themselves that enable these professionals to detect and avoid the spread of misinformation pieces of news. It is based on a methodological triangulation. Quantitative data have been obtained through an online questionnaire administered to a sample of 200 students enrolled in the academic year (2019/2020) in the degrees of the area of Journalism. And through two focus groups with students of these degrees we have qualitatively approached the opinion of future journalists about fake news. Despite the fact that almost three out of every four students surveyed state that they know how to distinguish a false news from a true one and more than half consider that the teachers have provided them with tools to fight against misinformation, $97.6 \%$ of students consider important to enhance media literacy in the university classrooms.
\end{abstract}

KEYWORDS: Journalism-Media Literacy-Fake News-Disinformation-Spanish Undergraduate

\title{
IMPACTO DAS FAKE NEWS NO JORNALISMO E NA COMUNICAÇÃO AUDIOVISUAL DOS ESTUDANTES DA UNIVERSIDADE CARLOS III DE MADRID
}

\section{RESUMO}

No atual contexto midiático, há cada vez mais vozes que alertam sobre a desinformação, notícias falsas ou fake news e o impacto que estas têm nas redes sociais nos jovens. Saber em que grau os estudantes de Jornalismo detectam e interagem com esse tipo de informação é necessário para articular estratégias dentro dos próprios estudos que capacitem os futuros profissionais a detectar e evitar a disseminação de peças de desinformação. A partir de uma triangulação metodológica, foram obtidos dados quantitativos, através de um questionário online aplicado a uma amostra de 200 alunos matriculados no ano letivo (2019/2020) nas licenciaturas da área de Jornalismo. E por meio de dois grupos focais com alunos dessas licenciaturas, foram obtidos dados qualitativos sobre a opinião de futuros jornalistas sobre notícias falsas. Apesar de quase três em cada quatro alunos afirmarem saber distinguir uma história falsa de uma verdadeira e mais da metade considerar que os professores lhes forneceram ferramentas para lutar contra a desinformação, 97,6\% consideram importante promover os meios de comunicação alfabetização desde as salas de aula da universidade. 
PALAVRAS-CHAVE: Jornalismo- alfabetização midiática- notícias falsasdesinformação- estudantes universitários espanhóis.

\section{INTRODUCCIÓN}

En la historia del periodismo siempre han existido las "no noticias", las pseudonoticias y las desinformaciones (Burnam, 1975; Galdón, 1994). En el siglo XIX emergen las noticias falsas acompañadas del crecimiento de los periódicos y de las tecnologías de aquel entonces (Berkowitz \& Schwartz, 2016). En el siglo XX uno de los docufake universalmente conocido es la Guerra de los Mundos que Orson Wells, quien interpretó en el estudio de la emisora CBS junto a otros compañeros la noche del 30 de octubre de 1938, una lectura radiofónica que generó pánico durante varios minutos a millones de radioyentes estadounidenses que creían que Nueva York iba a ser invadida por los alienígenas.

En 2016 Oxford eligió como palabra del año el término posverdad (post-truth), definida como: "circunstancias en que los hechos objetivos influyen menos en la formación de la opinión pública que las referencias a emociones y a creencias personales". Su potencial no radica en el nivel de verdad que contienen sino en el poder de emocionar y reforzar las ideas previas que tienen ciudadanas y ciudadanos respecto a un tema.

Sin embargo, existen también algunas aproximaciones teóricas más críticas hacia este concepto de posverdad que consideran que se culpa a receptores de algo que escapa de su control, ya que más bien estaríamos ante un fenómeno que se convierte en una "estrategia al servicio del mantenimiento de una determinada retórica de la verdad" cuando la objetividad o los hechos "no surgen de experiencias directas", sino que se trata de "fragmentos discursivos que contienen el sesgo del emisor" que las narra o el enfoque del medio para el que se trabaja (Carrera, 2020, p. 81).

En 2017 el diccionario inglés Collins elige el término fake news como palabra del año y la define como "información falsa, a menudo sensacional, difundida bajo el disfraz de la información de noticias".

\subsection{Las fake news en el periodismo}

El fenómeno de las fake news está hoy mucho más presente que nunca gracias, en gran medida, a las redes sociales. En el anterior paradigma comunicativo los medios de comunicación tenían la exclusividad de "empaquetar sus informaciones" (Amorós, 2018, p. 159) y distribuirlas al resto del mundo. Ahora se ha sido sustituido por una esfera mediática en la que cualquiera puede crear informaciones, viralizarlas o compartirlas con miles de personas en cuestión de segundos a través de sus plataformas sociales.

Las fake news representan un problema para periodistas, cuya credibilidad en los últimos años se ha visto disminuida a consecuencia de este fenómeno, y un reto para las autoridades políticas que exigen una regulación sobre el tema. Las elecciones 
estadounidenses de 2016 fueron el trampolín para este tipo de desinformaciones que se viralizaron y compartieron a través de las redes sociales con mentiras disfrazadas de noticias serias por distintas candidaturas (Amorós, 2018; Magallón, 2019; Rodríguez-Andrés, 2017). Internet y las redes sociales se han alzado como los canales más eficaces para la difusión de forma indiscriminada de este tipo de contenidos (Molina y Magallón, 2019; Salaverría, Buslón et al., 2020).

Desde las instituciones y organizaciones internacionales también crece la preocupación por las noticias falsas. Para intentar frenar la propagación de estas noticias falsas han surgido en la actualidad plataformas de fact-checking o verificación de la información, donde trabajan periodistas cuyo principal objetivo es detectar bulos o desinformaciones y desmentirlos a partir de la verificación de contenidos y el contraste de fuentes. Este trabajo es independiente de intenciones políticas (Elizabeth, 2014) y tiene su origen en la tradición de la prensa estadounidense, ya que medios como el Time o The New Yoker tenían sus propios departamentos para el control de los procesos de edición y verificación de sus contenidos (Salaverría, Buslón et al., 2020).

En España las principales plataformas de verificación acreditadas por la red internacional de fact-checkers International Fact-Checking Network (IFCN) son: Maldita.es, Newtral y EFE Verifica (Ufarte-Ruiz, Anzera \& Murcia-Verdú, 2020; Vizoso \& Vázquez-Herrero, 2019). A pesar de que las funciones que se desarrollan desde estas plataformas son inherentes al quehacer periodístico la rapidez en la propagación de fake news en redes sociales ha hecho que sirvan de apoyo a la ciudadanía y, en ocasiones, a los propios medios de comunicación. El papel de los periodistas que trabajan en ellos es esencial: "los ciudadanos acuden a ellos cuando necesitan comprender lo que sucede" (Herrero, 2020). Es importante que los futuros periodistas conozcan el método de trabajo y tengan herramientas suficientes para detectar las noticias falsas. La base fundamental para ello se basa en la alfabetización mediática.

\subsection{Alfabetización mediática frente a las fake news}

En el contexto digital actual la proliferación de noticias falsas y la vulnerabilidad de una sociedad escasamente alfabetizada mediáticamente, -en cuanto a la producción de mensajes propios y la interacción con otros ajenos establecida por los indicadores de Ferrés y Piscitelli, (2012)-, ha evidenciado la importancia, más que nunca, de la educación mediática. Una educación que va más allá del mero uso instrumental de las nuevas tecnologías y redes sociales y apuesta por el conocimiento y uso crítico de las mismas (Gutiérrez Martín, 2008; Lotero, Romero et al., 2018). Según un estudio con estudiantes de Periodismo de la Universidad del País Vasco las redes sociales son la principal vía de procedencia de las noticias falsas siendo Facebook la red social más citada (Mendiguren et al., 2020, pp. 176-177).

La Comisión Europea propuso en 2018 una serie de iniciativas para frenar las noticias falsas entre las que estaba promover la alfabetización mediática e informacional para contrarrestar la desinformación. 
Numerosos trabajos basados en metodologías y experiencias innovadoras educomunicativas, como los recopilados en la obra coordinada por Marfil-Carmona, Osuna-Acedo y et al. (2018) demuestran el esfuerzo docente que se está llevando a cabo en ámbitos como el universitario, objeto de este estudio, aunque aún no esté reglado de forma general en toda la universidad. En este sentido algunos frutos proceden de iniciativas y esfuerzos de los propios profesores, o de algunos Grados de universidades concretas, como la asignatura de "Comunicación y Educación" en el Grado de Pedagogía y en el de Educación Social de la UNED. Y en ámbito del periodismo la Universidad de Málaga, por mencionar otro caso, ofrece en su Grado como asignatura optativa de 6 créditos "Alfabetización mediática". Esta escasa presencia ya era subrayada en el trabajo de López y Aguaded (2015) que señalaban en su investigación de la docencia sobre alfabetización mediática en las facultades de Educación y Comunicación, que en la enseñanza superior:

No existen instrumentos que hayan validado los procesos de alfabetización entre los discentes universitarios, así como la motivación y formación del profesorado o las referencias a la educación mediática en los manuales bibliográficos más empleados en el proceso de docencia (2015, p. 189).

En cualquier caso, lo que parece evidente es que la formación del profesorado es un desafío clave en el proceso de alfabetización mediática, como señalan Alcolea-Díaz, Reig et al. (2020, p. 106) en su análisis sobre la presencia en el currículo del profesorado de Alfabetización Mediática e Informacional (AMI): “La propagación de la desinformación y las "fake news» plantea un severo desafío a los sistemas educativos, siendo claves el desarrollo del pensamiento crítico y las competencias analíticas para una intervención educativa exitosa".

Fernández-García (2017) presenta diferentes proyectos de educación mediática concluyendo que esta alfabetización es más necesaria que nunca y abogando por la transparencia de los medios y la participación de las audiencias: "Si las nuevas generaciones obtienen su información de redes sociales y otros recursos en línea, deben aprender a decodificar lo que leen" (2017, p. 75).

En los estudios de Periodismo y Comunicación esta alfabetización mediática alude también sin duda a la propia alfabetización periodística. En la investigación de PérezTornero et al., (2018) sobre cómo afrontar las que denominan "noticias falseadas", dada su intencionalidad-, apuestan por la transparencia, la calidad informativa, la participación y colaboración de los ciudadanos: "Existen diversas iniciativas de alfabetización mediática que se mueven entre el reducido campo del fact-checking hasta el más amplio del pensamiento crítico y de los valores humanistas y cívicos" (p. 231).

En el mismo sentido, Aguaded y Romero-Rodríguez (2015) abogan por la necesidad de integrar la alfabetización mediática e informacional, como ya señaló la UNESCO en 2012, mediante la reformulación de los medios desde las audiencias. 
Esta alfabetización afecta también al perfil profesional actual del periodista, que va más allá de las meras competencias tecnológicas, que demanda nuevas competencias para la comunicación en red. Esta falta de adecuación entre la formación académica de los periodistas y las demandas de las ofertas laborales han sido subrayadas en diferentes estudios (Álvarez-Flores, Núñez-Gómez y Rodríguez Crespo, 2017; MartaLazo, González-Aldea et al., 2018; Pérez-Serrano, Rodríguez-Barba et al., 2015). La alfabetización digital en el periodismo supone, en palabras de Usher (2019), convertirnos en "traductores profesionales", "intérpretes" de la información de modo que hoy en día incluso "es mucho más importante estar digitalmente alfabetizados que desarrollar cualquier otra habilidad periodística".

Sin embargo, no hay que olvidar que la tecnología es sólo la herramienta, que evoluciona rápidamente, y lo que sigue permaneciendo son los fundamentos: "Para saber filtrar y contrastar los contenidos de redes sociales sería importante abogar por la Educomunicación como medio para potenciar el 'humanismo digital' donde el sentido común prepondere sobre la infobasura" (Marta-Lazo, González-Aldea et al., 2018, p. 148). En el mismo sentido, de una formación que no olvida lo esencial del periodismo, como es la comprobación y contraste de informaciones, Bandrés, Badillo et al. (2018, p. 167) piden un compromiso a las universidades de los grados de Periodismo y Comunicación y a sus docentes para "fomentar el exceso de celo a la hora de comprobar y verificar la veracidad de las informaciones".

En definitiva, parece que la transparencia de los medios, el compromiso con la verificación de las informaciones, -tanto por parte de los periodistas como de sus empresas-, así como la participación de las audiencias resultan esenciales en la lucha contra las fake news desde la alfabetización mediática.

\section{OBJETIVOS}

La importancia y el creciente interés por las fake news se manifiesta en la producción científica generada alrededor del término. En los últimos años el número de trabajos académicos publicados en Scopus y WoS sobre fake news se ha incrementado de forma significativa. En 2017 y 2018 se han registrado 126 y 156 artículos respectivamente en estas bases de datos, frente a los 7 artículos sobre el tema localizados en 2016 (Parra \& Oliveira, 2018). Sin embargo, apenas hay estudios concretos -más allá del reciente de Mendiguren et al. (2020) en el País Vasco- sobre el impacto de estas fake news en estudiantes de Periodismo españoles, en futuros profesionales de la información a quienes esta cuestión afecta de pleno.

Conocer por tanto en qué grado estudiantes de Periodismo detectan e interactúan con este tipo de información es necesario y relevante para articular estrategias dentro de los propios estudios que capaciten al alumnado para detectar y evitar la propagación de piezas desinformativas. La hipótesis de partida apunta a que los hábitos de consumo mediáticos de los jóvenes en general favorecen la propagación de noticias falsas y aunque estudiantes de Periodismo y Comunicación pueden distinguir más fácilmente una noticia verdadera de una falsa, crean, viralizan y/o 
comparten fake news porque a pesar de la formación específica de su carrera suspenden en alfabetización mediática.

Por ello, esta investigación tiene por objeto conocer cómo estudiantes de Periodismo y Comunicación interaccionan con las fake news así como medir el grado de satisfacción con la alfabetización mediática y las herramientas que reciben en sus estudios universitarios para combatir estas noticias falsas.

\section{METODOLOGÍA}

Para abordar el objeto de estudio se ha llevado a cabo una triangulación metodológica combinando técnicas de orden cualitativo (revisión bibliográfica y focus group) y cuantitativo (encuestas). Esta investigación se centra en receptoras/es y adopta la vertiente culturalista de la investigación de audiencias que considera que "el mismo proceso del uso de los media como conjunto de prácticas y la manera en que se manifiestan constituyen el principal objeto de interés" (McQuail, 2000, p. 452). Se trata de conocer cómo los estudiantes de Periodismo reciben y consumen la información e interaccionan con ella. El trabajo sigue una estrategia exploratoriodescriptiva que incluye descripción, registros, análisis e interpretaciones.

El universo está formado por estudiantes matriculados durante el curso 19/20 en los Grados del área de Periodismo ofertados en la Facultad de Humanidades, Documentación y Comunicación de la Universidad Carlos III de Madrid. En total son 1.231 matriculados (ver tabla 1). La muestra estudiada para un nivel de confianza del $90 \%$ y un error muestral del $+/-5 \%$ es de 190 estudiantes, y finalmente fueron encuestados 202 estudiantes. La tabla fue elaborada a partir de los datos publicados por el servicio de calidad de la Universidad Carlos III de Madrid. Disponibles en https://www.uc3m.es/cld/matricula

Tabla 1: Estudiantes matriculados curso 2019/2020

\begin{tabular}{|l|l|}
\hline \multicolumn{2}{|c|}{$\begin{array}{c}\text { Matriculados área Periodismo UC3M } \\
\text { Curso 2019/2020 }\end{array}$} \\
\hline Grado en Periodismo & 424 \\
\hline Doble grado en Periodismo y Comunicación Audiovisual & 582 \\
\hline Doble grado de Periodismo y Humanidades & 225 \\
\hline Total & $\mathbf{1 2 3 1}$ \\
\hline
\end{tabular}

Fuente: elaboración propia 
Para la selección de la muestra hemos llevado a cabo un muestreo no probabilístico. Docentes de distintas asignaturas y cursos de los diferentes grados subieron en el aula virtual de la universidad un enlace con acceso a la encuesta.

Por último, con el objetivo de interpretar y enriquecer la discusión de los datos obtenidos a partir de la encuesta, se han desarrollado dos focus group, una técnica desarrollada en el campo de la investigación en comunicación de masas (Eiroa y Barranquero, 2018).

El focus group fue online a través de la plataforma Google Meet y se desarrollaron dos grupos en dos días distintos. Cada grupo estaba constituido por 6 personas (mitad mujeres y mitad hombres), estudiantes de primero del Doble Grado en Periodismo y Comunicación Audiovisual (PERYCOM) y de primero y cuarto del Grado en Periodismo. Para la dinamización del mismo se utilizó un guión que recogía algunos de los bloques temáticos más importante de la investigación (consumo mediático, fake news y alfabetización mediática) y se plantearon de forma abierta cuestiones que recogían algunos de los resultados más significativos que se habían recogido en la encuesta.

\section{RESULTADOS}

\subsection{Hábitos mediáticos de los estudiantes de Periodismo y/o Comunicación}

Un $63,90 \%$ de los estudiantes del estudio indican que consultan los medios de comunicación una vez al día, y un $27,70 \%$ señala acceder a los mismos varias veces. Casi un 6\% indican que consultan la información cada tres o cuatro días, y sólo un $2,5 \%$ de los encuestados señala consultar las noticias una vez a la semana o nunca.

En cuanto a los medios más consultados para mantenerse informadas/os (gráfico 1), la mayoría señala las redes sociales $(94 \%)$ y los periódicos digitales $(93,10 \%)$. Un $68,3 \%$ indica la televisión como principal medio de acceso a la información, y muy por detrás están el medio radiofónico (35,1\%) y los diarios en papel (31\%). En cuanto a la categoría "otros" el dato no es significativo (1\%) y señalan las newsletter o lo que comentan con su entorno. 


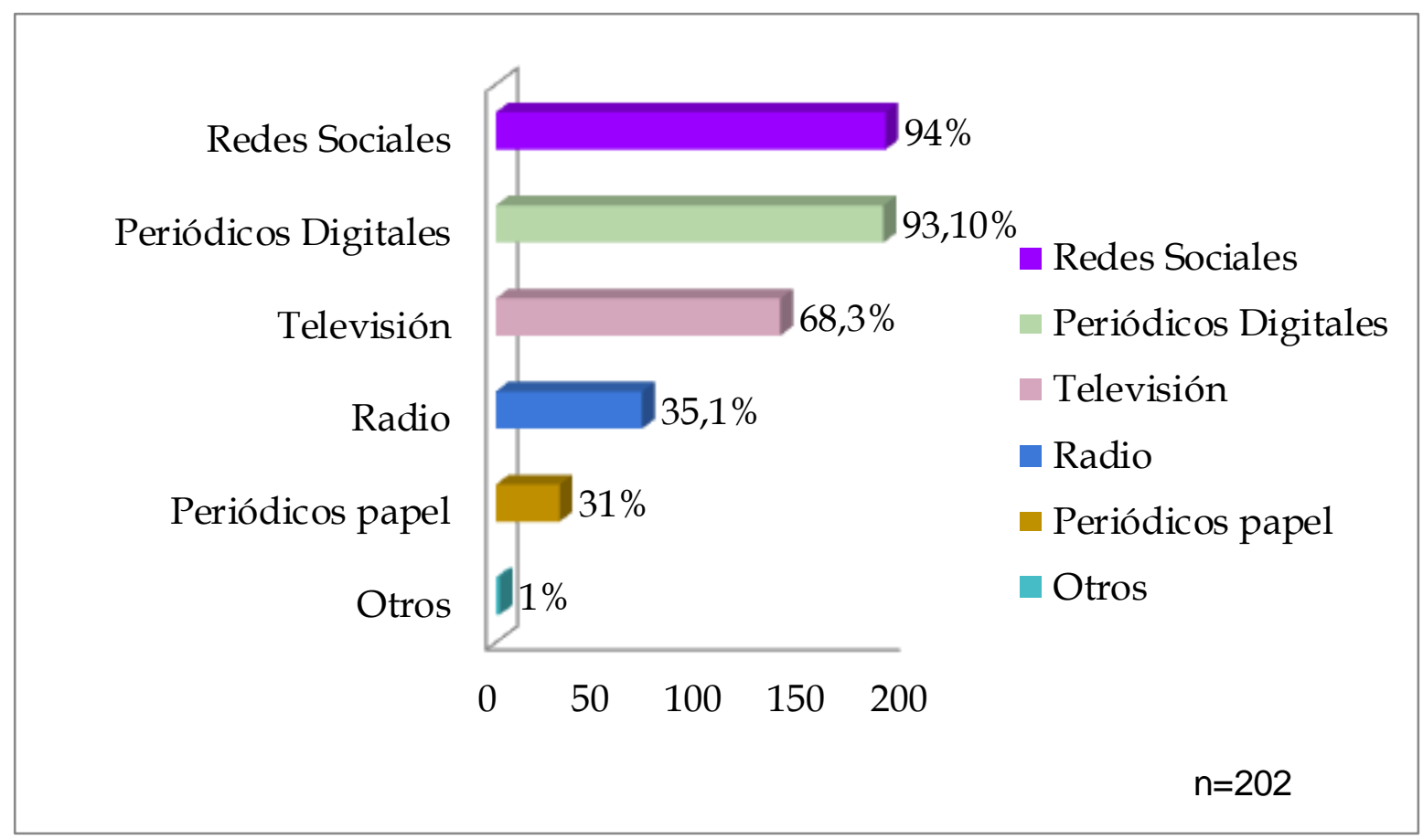

Gráfico 1: Principales medios de acceso a la información (respuesta múltiple)

Fuente: elaboración propia.

Consideran que informarse de este modo no supone necesariammente más exposición a las noticias falsas.

"Yo creo que es lo contrario, porque nosotros tenemos más consciencia precisamente porque utilizamos las redes sociales. No todo lo que se dice allí es verdad y tendemos a comprobar más la información. Sin embargo, nuestros padres, su generación, como las usan menos, son menos nativos en ese aspecto, todas las cosas que les digas se las creen. A mí por el grupo de la familia siempre me llegan este tipo de bulos" (Estudiante 1, Doble Grado, $4^{\circ}$ curso).

No obstante, el hecho de estar más acostumbrados al uso de redes sociales puede ser para muchos un arma de doble filo.

“Es verdad que los jóvenes estamos más acostumbrados a las redes y podemos verificar si una noticia es falsa o no mejor que los mayores, tenemos más herramientas. Pero también sabemos cómo difundirlas de una forma más rápida que nuestros padres" (Estudiante 2, Grado Periodismo, $1^{\circ}$ curso)

"Por un lado tenemos más criterio a la hora de diferenciar una noticia falsa, pero a la hora de compartir información muchas veces lo hacemos sin tener mucho cuidado" (Estudiante 3, Doble Grado, $1^{\circ}$ curso)

En cuanto a la cuestión de cómo consumen las noticias (respuesta múltiple), se quería conocer el nivel de profundización en sus lecturas. En este sentido, un $69,30 \%$ de los 
estudiantes señala leer sólo los titulares y la entradilla. Y más de la mitad señala leer tuits (55,90\%). Sólo el 50,50\% lee la noticia completa. Un 29,20\% lee únicamente los titulares, y un $7 \%$ reconoce que depende del interés que le suscite la noticia, del contenido, lee únicamente el titular o avanza más allá del primer párrafo.

Los estudiantes reconocen que esta lectura superficial puede ser un problema a la hora de identificar noticias falsas:

“Muchas veces el método más rápido para informarte es a través de las redes sociales o con las aplicaciones de algún periódico. Te salta la notificación y solo con el resumen que te pone ahí te piensas que estás bien informado pero deberías leer la noticia al completo, incluso contrastarla con otros periódicos" (Estudiante 4, Grado Periodismo, $1^{\circ}$ curso)

"Nos fiamos mucho de los titulares. De hecho, si un titular te llama la atención muchas veces haces una captura de pantalla y la subes a redes sociales y a lo mejor lo que dice ese titular tiene muchos más matices en el resto de la noticia que en el titular no se ven. $Y$ esto se puede viralizar sin haber comprendido la noticia" (Estudiante 7, Grado Periodismo, $1^{\circ}$ curso)

La cultura de la lo inmediato en la que viven y las prácticas de los medios con titulares llamativos son mencionados como principales razones a la hora de explicar ese consumo superficial de noticias.

"Creo que también es parte de la cultura que tenemos en la actualidad, de lo inmediato. $Y$ esa cultura afecta a la hora de informarnos. Si vemos una noticia muy larga la gente lee el titular y ya está. Y otras veces pasa que lo que sigue no tienen nada que ver" (Estudiante 10, Doble Grado, $3^{\circ}$ curso)

“Los titulares están hechos sólo para llamar la atención, para que te metas y que el medio cobre por los anuncios. Les da igual si lees la noticia entera o no y muchas veces el titular no tiene que ver con la noticia" (Estudiante 12, Grado Periodismo, $3^{\circ}$ curso)

“No sé si el problema está tanto en la noticia o en nosotros. A la gente le gusta enterarse de lo que pasa pero no saber qué pasa. Es lo que tiene leer solo el titular y la entradilla, te enteras pero no sabes" (Estudiante 5, Grado Periodismo, $4^{\circ}$ curso) 


\subsection{Conocimiento de las fake news}

El 70,80\%, casi tres de cada cuatro estudiantes, afirman saber distinguir una noticia falsa de una verdadera. Más significativo y quizás preocupante es que un 29,20\% que señala abiertamente no saber distinguir una noticia real de otra falsa.

En cuanto a qué elementos tienen en cuenta los estudiantes para saber que la noticia es falsa (gráfico 2) el $85,60 \%$ señala que se fija en el medio que lo publica, un $55 \%$ en la naturaleza de las fuentes que se citan en la noticia, un 53,50\% la autoría de la información y la fecha en la que ha sido publicado, un 40,10\% en el número de fuentes que aparecen en la pieza informativa $\mathrm{y}$, por último, con un dato menos significativo un $13,90 \%$ de los estudiantes encuestados señala que se fija en el número de veces que la noticia ha sido compartida. Durante los últimos meses redes sociales como Whatsapp han limitado el número de veces que se puede reenviar un mensaje a los grupos o contactos. Parece que aquellos mensajes que son reenviados con mucha frecuencia tienen más probabilidades de contener bulos.

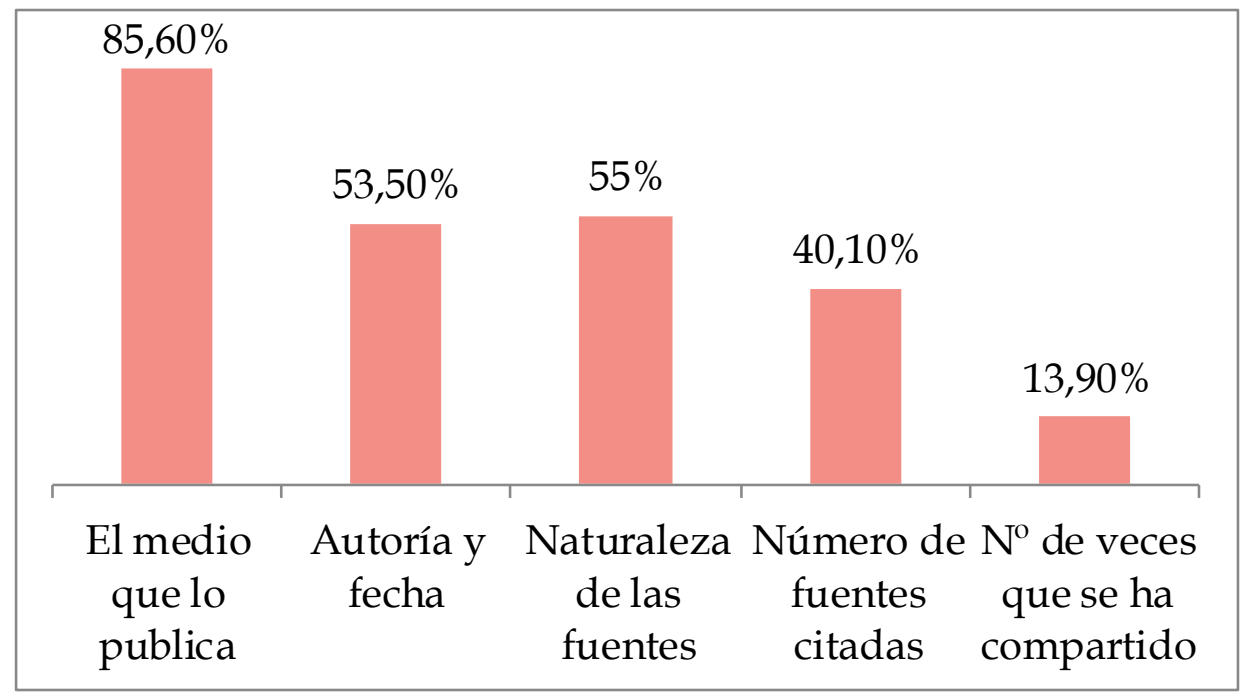

Gráfico 2. En qué se fijan los estudiantes para saber si una noticia es falsa (respuesta múltiple)

Fuente: elaboración propia.

Siendo la clave, para casi el $86 \%$ de los encuestados, el medio de comunicación que lo publica acuden a medios tradicionales para verificar las informaciones en caso de duda porque los consideran más fiables.

"Me pasa que a veces cuando leo una noticia que me suena rara, que puede ser fake news, lo primero que hago es ver qué medios lo han publicado. Y muchas veces me sale la noticia en varios medios, pero todavía no le doy la credibilidad suficiente si medios grandes como El País, ABC, La Vanguardia y demás no la han publicado" (Estudiante 6, Grado Periodismo, 4 curso) 
"Los medios tradicionales son más creíbles, pero todos son susceptibles de caer en las fake news" (Estudiante 1, Doble Grado, $4^{\circ}$ curso)

Cuando se pregunta a los estudiantes a través de qué medio han recibido más fake news, el 45,50\% señala que Whatsapp es el principal canal, seguido de un $36,60 \%$ que apuntan a redes sociales como Facebook o Twitter. Un dato preocupante si se tiene en cuenta que las redes sociales son el medio por el que más estudiantes se mantiene informado. Por último, un 5\% señaló otras como Instagram o confidenciales.

Aunque Whastapp sea el medio por el que más reciben fake news, para los estudiantes resulta sin embargo de entrada el menos fiable.

“Estoy de acuerdo, tendemos a creernos más lo audiovisual pero cuando te llega una imagen por WhatsApp siempre es mejor esperar un poco a saber si es verdad o con herramientas de verificación como pueden ser Maldito Bulo o Newtral" (Estudiante 10, Doble Grado, $3^{\circ}$ curso)

El $84,20 \%$ de los estudiantes considera que los ciudadanos son los mayores generadores de fake news seguido de un $44,60 \%$ que opina que los políticos son los que más fake news crean y un $37,60 \%$ señala a los medios como los principales creadores de noticias falsas.

Para los estudiantes el hecho de que los medios no sean los principales creadores de noticias falsas y aparezcan en tercer lugar no disminuye la gravedad del hecho.

"A los medios a ninguno le conviene difundir fake news por la fama que pueda coger y que pierda toda la credibilidad. Su papel debería ser ponerles fin, desmentirlas, dar información veraz con la que podamos contrastarlas" (Estudiante 4, Grado Periodismo, $1^{\circ}$ curso)

“Las fake news las sacan los políticos, los ciudadanos... pero quien las difunde es el medio de comunicación muchas veces. Yo responsabilizo más a los medios que a los ciudadanos o a los políticos" (Estudiante 5, Grado Periodismo, $4^{\circ}$ curso)

En cuanto a los temas de fake news que más se comparten se encuentran: temas sociales $(44,70 \%)$, política $(23,50 \%)$, deporte y salud $(11,80 \%)$. De forma menos significativa estaría la ciencia $(2,40 \%)$.

Más de la mitad de los estudiantes que compartieron fake news señalan que se enteraron de que la noticia era falsa (gráfico 3) a través de los medios de comunicación $(28,20 \%)$ y las propias redes sociales $(28,20 \%)$ y un $21,20 \%$ señala a familiares y amigos a la hora de detectar que la noticia era falsa.

Por otro lado, sólo un $16,50 \%$ de los encuestados señalan que se enteraron de la falsedad de la noticia por plataformas de verificación, como Newtral o Maldita. El resto de encuestados $(6 \%)$ no recuerda dónde se enteraron. 


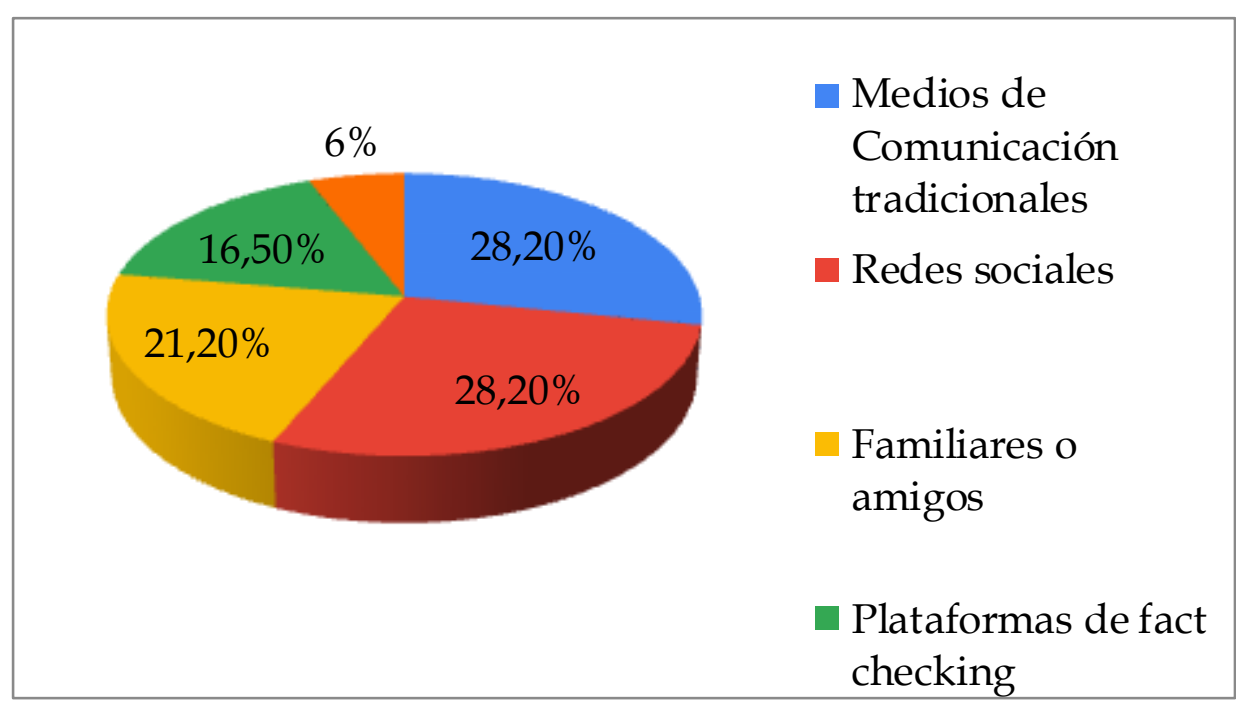

Gráfico 3. ¿Cómo se enteran de que la noticia era falsa?

Fuente: elaboración propia.

\subsection{Alfabetización mediática en la universidad}

El $97,70 \%$ de los estudiantes consideran importante potenciar la alfabetización mediática en la universidad. Los estudiantes consideran que la alfabetización mediática es necesaria, pero no sólo en los estudios de Periodismo y Comunicación, sino en todas las carreras e incluso ya desde niveles pre-universitarios, en edades mucho más tempranas.

“Debe ser antes, desde primaria o secundaria, como asignatura que explique las repercusiones de difundir fake news. Se es muy blando judicialmente con estos temas. Si hubiera ejemplos judiciales más fuertes la gente se lo pensaría antes de difundir una noticia falsa" (Estudiante 12, Grado Periodismo, $3^{\circ}$ curso)

“Yo también estoy de acuerdo. No debería ser una cosa sólo de las carreras universitarias ligadas a los medios de comunicación, sino una labor que se lleva a cabo en las casas y en edades más tempranas. Me parece una labor muy importante porque todo el mundo dispone ya de redes sociales y puedes difundir estas noticias" (Estudiante 4, Grado Periodismo, $1^{\circ}$ curso)

No obstante, los estudiantes creen que esta alfabetización mediática es especialmente relevante y necesaria en el caso de los comunicadores.

"Si nosotros vamos a ser fuente fiable para los demás ya no sólo en el mundo profesional, sino para amigos, familia... porque como estudiamos Periodismo acuden a nosotros para preguntarnos cosas sobre estos temas de fake news, creo que debemos estar más concienciados y saber más sobre el tema" (Estudiante 11, Grado Periodismo, $3^{\circ}$ curso) 
"La mayoría de profesores hacen mucho hincapié en fuentes y veracidad. Rompo una lanza a nuestro favor porque creo que tenemos más cuidado con las fake newws. Los amigos de mi edad, que en alfabetización en redes sociales tienen el mismo nivel, es cierto que prestan menos atención en comprobar fuentes antes de compartir algo que un estudiante de Periodismo como yo misma" (Estudiante 10, Doble Grado, $3^{\circ}$ curso)

Son muy pocos, no obstante, los estudiantes que entienden la alfabetización mediática en su pleno sentido, no sólo como una formación instrumental o técnica en el manejo de la tecnología, sino en su significado más amplio que implica las consecuencias de su mal uso y la responsabilidad de servicio público.

"Más que educación en redes sociales, creo que es necesaria una educación ética. El problema no es muchas veces saber si es mentira o no la noticia que se va a publicar, sino saber si merece la pena publicar algo falso por los beneficios que pueda suponer o prefiero no publicarla y ser un buen periodista" (Estudiante 3, Doble Grado, $1^{\circ}$ curso)

Un 70,45\% está satisfecho con los recursos o herramientas que su profesorado le ha facilitado para detectar fake news. Mientras que un $29,55 \%$ no las considera suficientes. Entre las herramientas o recursos que los docentes les han facilitado señalan principalmente: Claves y técnicas para saber identificar una noticia falsa, herramientas para verificar información en internet, ejemplos de verificación de contenidos, periodismo de datos y charlas impartidas por profesionales de la verificación.

En la medida en que los medios dedicados en España al fact-cheking han aparecido sobre todo en los últimos dos años (Newtral 2018, o Maldita.es en 2018), los estudiantes de últimos cursos muestran opiniones diferentes en cuanto a herramientas proporcionadas en la carrera, frente a los estudiantes de primeros cursos.

“Estoy en primero de carrera, pero estoy satisfecho con la formación para poder verificar las noticias. No conocía Newtral o Maldita por ejemplo, y son herramientas fundamentales para desmentir noticias. Pero van a seguir existiendo de forma inevitable y está en cada uno difundirlas, incluso crearlas, o no" (Estudiante 2, Grado Periodismo, $1^{\circ}$ curso)

“En el plan actual sí nos enseñan cómo funcionan Maldita, Newtral... y nos dan clase utilizando las propias herramientas que aparecen en esas páginas para desmentir los bulos. Se está cambiando la forma de enseñar Periodismo en la universidades" (Estudiante 3, Doble Grado, $1^{\circ}$ curso)

Sin embargo, los estudiantes de último curso muestran su desacuerdo con las herramientas facilitadas. 
“Acabo este año. Hay buenas intenciones porque te hacen mucho hincapié en las fuentes y la veracidad como conceptos, pero no profundizan más allá. Me gustaría que dieran algo de esperanza o herramientas para hacerle frente, porque creo que la sociedad ya se ha rendido" (Estudiante 1, Doble Grado, $4^{\circ}$ curso)

“Descubrí estas herramientas y algunas cosas más en mis prácticas. Me sentía perdidísima y había pasado una carrera. Creo que más que la asignatura y las herramientas es según los profesores con los que te toquen" (Estudiante 5, Grado Periodismo, $4^{\circ}$ curso)

Estos testimonios ponen de relieve la urgencia de esa educación mediática, que más allá de estas herramientas de fact checking, les hagan comprender que la base de todo está en los fundamentos y en la calidad del periodismo.

\section{DISCUSIÓN}

Los hábitos mediáticos de los estudiantes de Periodismo y/o Comunicación muestran un bajo nivel de actualización informativa frente al que cabría esperar de estudiantes de Periodismo y Comunicación. Actualmente para estar informado hay que consultar la información más de una vez al día.

El hecho de que acudan sobre todo a redes sociales y periódicos digitales para mantenerse informados está en consonancia con los hábitos de la generación analizada, nacida en plena emergencia de las redes sociales. Pese a que las redes sociales son caldo de cultivo de fake news los jóvenes encuestados creen que por el hecho de informarse más a través de redes sociales y medios digitales no tienen necesariamente que tener más exposición a las noticias falsas. No obstante, reconocen que la rapidez y la falta de comprobación hace que muchas veces ellos viralicen de forma más rápida que otras generaciones, como sus padres, esas noticias falsas.

Casi tres de cada cuatro encuestados confiesan que leen sólo los titulares y la entradilla de las noticias. Se trata de dos de los principales elementos que configuran la estructura externa de la noticia y dan información de lo que ocurre, pero el nivel de profundización en el tema es escaso. A ello se une el que casi un 56\% lee tuits, lo que es coherente con el uso de redes sociales mencionado por ellos mismos para estar actualizados donde la mayoría señalaba las redes sociales como el principal medio que usaban para acceder a las noticias.

Esta lectura superficial de las noticias puede ser un problema a la hora de estar bien informados y no caer en las fake news que mediante titulares llamativos consiguen la rápida reacción a la hora de compartirlos sin contrastar la información recibida. Para algunos la responsabilidad de no hacerlo está en ellos mismos, influenciados como viven en la cultura del consumo rápido y la inmediatez, pero también responsabilizan a los medios y sus políticas de clickbait, o anzuelos para pinchar en titulares llamativos. 
En cuanto al conocimiento de las fake news los resultados van un poco por delante de la percepción que tenía la población general cuando emergía el tema de las fake news en España, donde el $60 \%$ de los españoles creía que sabía detectar una noticia falsa (Manzanero, 2017). Pero lo más significativo, y quizás preocupante, es que casi un tercio señala abiertamente no saber distinguir una noticia real de otra falsa, un porcentaje que no es tan bajo si tenemos en cuenta que estos estudiantes de periodismo deben prepararse para trabajar en un contexto en el que verificar las informaciones es esencial en la lucha contra las fake news.

Siendo la clave para identificar una noticia falsa el medio de comunicación para casi el $86 \%$ de los encuestados cabría preguntarse si consideran que hay medios más y menos creíbles, o si los medios tradicionales tienen más credibilidad, son más fiables que los nativos digitales o al contrario. Los medios tradicionales resultan más fiables según los estudiantes, lo que choca con el hecho de que luego no sean los elegidos a la hora de consumir información.

Parece evidente que hay falta de conciencia colectiva de las repercusiones de la difusión de noticias falsas, como muestra el dato de que los estudiantes consideren a los ciudadanos como los mayores generadores de fake news. Es en este ámbito donde el periodismo debe cobrar protagonismo desmintiendo las informaciones falsas.

Los temas sociales y la política son para los estudiantes los principales focos de noticias falsas. Estos resultados coinciden también con los extraídos de una investigación realizada con estudiantes de Periodismo en el País Vasco que consideran que son los temas sociales y políticos donde más abundan las fake news (Mendigueren et al., 2020, p. 177), si bien los estudiantes vascos sitúan la política en primer lugar.

Las redes sociales y los ciudadanos se convierten a la vez en agentes de acceso a la información y a la desinformación, según los propios estudiantes, al señalarlos como las principales vías por las que supieron que una información era falsa. El que sólo un $16,5 \%$ de los encuestados se enterasen de la falsedad de la noticia por plataformas de verificación, como Newtral o Maldita resulta significativo puesto que al ser estudiantes de Periodismo cabría esperar que para contrastar si una noticia es un bulo, o no, acudieran a estas plataformas.

Teniendo en cuenta los resultados descritos los estudiantes perciben que necesitan más medios o herramientas para detectar bulos o noticias falsas, y en este sentido casi uno de cada tres no considera suficientes las herramientas y recursos proporcionados por sus profesores. Por ello, casi el $98 \%$ afirma que debe potenciarse la alfabetización mediática en la universidad. Esto coincide con los resultados de la investigación con estudiantes de Periodismo del País Vasco que apuntaba que "el 91\%, cree que sería interesante que se implantaran cursos formativos en las escuelas para enseñar a distinguir la información fiable de la que no lo es" (Mendiguren et. al, 2020, p. 179). 


\section{CONCLUSIONES}

Los resultados de esta investigación muestran algunos datos preocupantes en cuanto al modo en que los estudiantes de periodismo y comunicación de la Universidad Carlos III de Madrid detectan e interactúan con las fake news. Los hábitos de consumo mediático de estos jóvenes, principalmente redes sociales y medios digitales $(90 \%)$, no suponen necesariamente en su opinión una mayor exposición a las noticias falsas, y señalan que pueden verificar si una noticia es falsa o no mejor que las personas mayores, y tienen más herramientas. De hecho, casi tres de cada cuatro estudiantes afirman saber distinguir una noticia falsa de una verdadera. Sin embargo, a pesar de la formación específica de su carrera, sí reconocen que las viralizan y las comparten.

Intentando comprender la razón por la que comparten esa información falsa detectamos que el modo de consumir las noticias influye. Sólo la mitad de los estudiantes encuestados señala leer la noticia completa y el 35\% sólo lee los titulares. Aunque culpan a los medios por sus prácticas de clickbait, o anzuelos para pinchar en titulares llamativos, reconocen que no profundizar en las noticias les lleva a compartir noticias falsas.

Whatsapp es la red por la que llegan más fake news según los estudiantes, seguida de Facebook y Twitter. Las redes sociales son las plataformas en las que más horas pasan los jóvenes y esta exposición masiva a las redes explicaría porque son también los canales desde donde más bulos o desinformaciones reciben.

Los medios tradicionales, las grandes cabeceras, son su referencia a la hora de comprobar posibles noticias falsas y en este sentido el $85,60 \%$ señala que se fija en primer lugar el medio que lo publica y también en la autoría de la información, por lo que la credibilidad del profesional, además del medio, resulta cada día más importante. Pese a las nuevas herramientas para detectar bulos y plataformas de verificación sólo un $16,50 \%$ acuden a ellas.

Un $29 \%$ asegura no distinguir una noticia real de una falsa. Se trata de algo especialmente grave si tenemos en cuenta que se trata de estudiantes de periodismo que en un futuro serán los responsables de crear y compartir la información y revela también que la formación del profesorado sigue siendo un desafío clave en el proceso de alfabetización mediática.

Aunque se está cambiando la forma de enseñar periodismo en las universidades, uno de cada cuatro estudiantes no está totalmente satisfecho y no considera suficientes los recursos o herramientas que su profesorado le ha facilitado para detectar fake news. De forma unánime creen que es importante potenciar la alfabetización mediática en la universidad en todas las carreras, e incluso ya desde niveles pre-universitarios.

Los resultados de la investigación en la Universidad Carlos III de Madrid muestran coincidencias con la llevada a cabo en el País Vasco, y pone de relieve la necesidad de 
más diagnósticos para diseñar estrategias formativas adecuadas dirigidas a los estudiantes de Periodismo y Comunicación.

Financiación: "Acción financiada por la Comunidad de Madrid a través de la línea de "Excelencia del Profesorado Universitario" del Convenio Plurianual con la UC3M (EPUC3MXX), en el marco del V PRICIT (V Plan Regional de Investigación Científica e Innovación Tecnológica)".

\section{REFERENCIAS}

Aguaded, I. y Romero-Rodríguez, L. M. (2015). Mediamorfosis y desinformación en la infoesfera: Alfabetización mediática, digital e informacional ante los cambios de hábitos de consumo informativo. Education in the Knowledge Society, 16 (1), 44-57. http://dx.doi.org/10.14201/eks2015161

Alcolea-Díaz, G.; Reig, R. y Mancinas-Chávez, R. (2020). Currículo de Alfabetización Mediática e Informacional de la UNESCO para profesores desde la perspectiva de la Estructura de la Información. Comunicar, 62, 103-114. https://doi.org/10.3916/C62-2020-09

Álvarez-Flores, E. P.; Núñez-Gómez, P. y Rodríguez Crespo, C. (2017). Adquisición y carencia académica de competencias tecnológicas ante una economía digital. Revista Latina de Comunicación Social, 72, 540-559. http://doi.org/10.4185/RLCS$\underline{2017-1178}$

Amorós, M. (2018). Fake news: la verdad de las noticias falsas. Plataforma Editorial.

Bandrés, E.; Badillo, M. E. y Ramos Antón, R. (2018). Noticias falsas, manipulación informativa e infopolución. En: C. Marta-Lazo (ed.) Calidad informativa en la era de la digitalización: fundamentos profesionales VS infopolución, 155-169. Editorial Dykinson.

Berkowitz, D., \& Schwartz, D. A. (2016). Miley, CNN and the onion: When fake news becomes realer than real. Journalism Practice, 10 (1), 1-17. http://doi.org/10.1080/17512786.2015.1006933

Burnam, T. (1975). The dictionary of misinformation. Thomas Y. Crowell.

Carrera, P. (2020). Basado en hechos reales. Cátedra.

Collins 2017 Word of the Year Shortlist (2017, 2 noviembre). Collins Dictionary. Sitio. https://www.collinsdictionary.com/word-lovers-blog/new/collins-2017-wordof-the-year-shortlist,396,HCB.html

Eiroa, M. y Barranquero, A. (2017). Métodos de Investigación en la comunicación y sus medios. Editorial Síntesis . 
Elizabeth, J. (2014). Who are you calling a fact checker? - American Press Institute. Sitio. $\quad$ https://www.americanpressinstitute.org/fact-checking-project/factcheckerdefinition/

Fernández-García, N. (2017). Fake news una oportunidad para la alfabetización mediática. Nueva sociedad, 269, pp. 66-77.

Ferrés, J. y Piscitelli, A. (2012). La competencia mediática: propuesta articulada de dimensiones e indicadores. Comunicar, 38, 75-82. https://doi.org/10.3916/C382012-02-08

Galdón, G. (1994). Desinformación: método, aspectos y soluciones. Pamplona: Eunsa.

Gutiérrez Martín, A. (2008). Educar para los medios en la era digital. Comunicar, XVI (31), 451-456.

Herrero, E. (2020). Periodismo en la era del Covid, un elogio al tratamiento de las fuentes. Telos. Repensar el mañana.

López, L. y Aguaded, M. 1. (2015). La docencia sobre alfabetización mediática en las facultades de Educación y Comunicación. Comunicar, 44, 187-195. https://doi.org/10.3916/C44-2015-20

Lotero, G.; Romero, L.M. y Pérez, M.A. (2018). Fact checking vs Fake news. Periodismo de confirmación como componente de la competencia mediática contra la desinformación. Index.comunicación. Revista científica en el ámbito de la Comunicación Aplicada, 8 (2), 295-316.

Magallón, R. (2019). Unfaking newws: cómo combatir la desinformación. Madrid: Ediciones Pirámide.

McQuail, D. (2000). Introducción a la teoría de la Comunicación de masas. Barcelona: Paidós Comunicación.

Manzanero, A. (2017, mayo). I Estudio sobre el Impacto de las Fake News en España. Sitio. https://d3vjcwm65af87t.cloudfront.net/novacdn/EstudioPescanova.pdf

Marfil-Carmona, R.; Osuna-Acedo, S. y González-Aldea, P. (eds.). Innovación y esfuerzo investigador en la educación mediática contemporánea. Sevilla: Egregius Ediciones.

Marta-Lazo, C.; González-Aldea, P. \& Herrero Curiel, E. (2018). Professional Skills and Profiles in Journalism Demanded by Companies: Analysis of Offers at LinkedIn and Infojobs. Communication \& Society, 31(4), 211-228.

Marta-Lazo, C.; González-Aldea, P. y Nogales-Bocio, A. I. (2018). Fundamentos profesionales y competencias de los nuevos informadores para la comunicación en 
red. En: C. Marta-Lazo (ed.), Calidad informativa en la era de la digitalización: fundamentos profesionales VS infopolución, (143-154). Editorial Dykinson.

Mendiguren, T.; Pérez Dasilva, J., \& Meso Ayerdi, K. (2020). Actitud ante las Fake News: Estudio del caso de los estudiantes de la Universidad del País Vasco. Revista de Comunicación, 19 (1), 171-184. https://dx.doi.org/10.26441/rc19.1-2020-a10

Molina, J. P. y Magallón, R. (2019). Procedimientos para verificar y desmontar informaciones falsas basadas en el discurso del odio. El caso de Maldita Migración. RAEIC, Revista de la Asociación Española de Investigación de la Comunicación, vol. 6, núm. 12, 95-122.

Oxford Languages. (2016). Oxford. https://languages.oup.com/word-of-theyear/2016/

Parra, P. y Oliveira, L. (2018). Fake news: una revisión sistemática de la literatura. Observatorio $\left(O B S^{*}\right)$, Special Issue, 54-78.

Pérez Serrano, M. J.; Rodríguez Barba, D. y Rodríguez Pallarés, M. (2015). Mercado de la Comunicación y estudiantes de Periodismo. Estructura de la demanda de perfiles profesionales. Revista Latina de Comunicación Social, 70, 209-229.

Pérez-Tornero, J. M.; Samy Tayie, S.; Tejedor, S. y Pulido, C. (2018). ¿Cómo afrontar las noticias falseadas mediante la alfabetización periodística: estado de la cuestión. Doxa Comunicación, 26, 211-235.

Rodríguez-Andrés, R. (2017). “Trump 2016: ¿presidente gracias a las redes sociales?”. Palabra Clave, 21(3), 831-859. DOI: https://doi.org/10.5294/pacla.2018.21.3.8

Salaverría, R.; Buslón, N.; López-Pan, F.; León, B.; López-Goñi, I. y Erviti, M. C. (2020). Desinformación en tiempos de pandemia: tipología de los bulos sobre la Covid-19. El profesional de la información, 29 (3). https://doi.org/10.3145/epi.2020.may.15

Ufarte-Ruiz, M. J.; Anzera, G. y Murcia-Verdú, F. J. (2020). Plataformas independientes de fact-checking en España e Italia. Características, organización y método. Revista Mediterránea de Comunicación, 11 (2), 1-17. https://www.doi.org/10.14198/MEDCOM2020.11.2.3

Usher, N. (2019). Alfabetización digital y futuro del periodismo. https:// scielo.conicyt.cl/scielo.php?pid=S0719$\underline{15292019000200216 \& \text { script }=\text { sci_arttext }}$

Vizoso, A. y Vázquez-Herrero, J. (2019). Plataformas de factchecking en español. Características, organización y método. Communication \& Society, 32, (1), 127-144. https://doi.org/10.15581/003.32.1.127-144 


\section{AUTORAS}

\section{Eva Herrero Curiel}

Doctora con mención Internacional en Investigación de Medios de Comunicación. Coordinadora y profesora de La Noticia Periodística en el Departamento de Comunicación de la Universidad Carlos III de Madrid. Es miembro del grupo de investigación Periodismo y Análisis Social: Evolución, Efectos y Tendencias (PASEET).

ORCID ID: https:/ / orcid.org/0000-0003-0801-2519

\section{Patricia González Aldea}

Doctora en Ciencias de la Información, (Departamento de Derecho Internacional Público y Relaciones Internacionales de la Facultad de Ciencias de la Información de la Universidad Complutense de Madrid, 1999). Profesora de Periodismo Internacional en el Departamento de Comunicación de la Universidad Carlos III de Madrid. Es miembro del grupo de investigación Periodismo y Análisis Social: Evolución, Efectos y Tendencias (PASEET).

ORCID ID: https:// orcid.org/0000-0002-1606-6034 